\title{
Pulsating Solitons in the Two-Dimensional Complex Swift-Hohenberg Equation
}

\author{
Aladji Kamagaté1,2, Alain-Brice Moubissi ${ }^{3}$ \\ ${ }^{1}$ Ecole Supérieure Africaine des Technologies d'Information et de Communication (ESATIC), Abidjan, Côte d'Ivoire \\ ${ }^{2}$ Agence Nationale de la Recherche, Paris, France \\ ${ }^{3}$ Université des Sciences et Techniques de Masuku, Franceville, Gabon \\ Email: alkamagate@gmail.com
}

How to cite this paper: Kamagaté, A. and Moubissi, A.-B. (2018) Pulsating Solitons in the Two-Dimensional Complex Swift-Hohenberg Equation. Journal of Applied Mathematics and Physics, 6, 2127-2141. https://doi.org/10.4236/jamp.2018.610179

Received: September 11, 2018

Accepted: October 26, 2018

Published: October 29, 2018

Copyright (c) 2018 by authors and Scientific Research Publishing Inc. This work is licensed under the Creative Commons Attribution International License (CC BY 4.0).

http://creativecommons.org/licenses/by/4.0/

\section{(c) (i) Open Access}

\begin{abstract}
In this paper, we performed an investigation of the dissipative solitons of the two-dimensional (2D) Complex Swift-Hohenberg equation (CSHE). Stationary to pulsating soliton bifurcation analysis of the 2D CSHE is displayed. The approach is based on the semi-analytical method of collective coordinate approach. This method is constructed on a reduction from an infinite-dimensional dynamical dissipative system to a finite-dimensional model. The reduced model helps to obtain approximately the boundaries between the stationary and pulsating solutions. We analyzed the dynamics and characteristics of the pulsating solitons. Then we obtained the bifurcation diagram for a definite range of the saturation of the Kerr nonlinearity values. This diagram reveals the effect of the saturation of the Kerr nonlinearity on the period pulsations. The results show that when the parameter of the saturation of the Kerr nonlinearity increases, one period pulsating soliton solution bifurcates to double period pulsations.
\end{abstract}

\section{Keywords}

Pulsating Solution, Dissipative Soliton, Spatio-Temporal, Collective Coordinate Approach, Ginzburg-Landau Equation, Complex Swift-Hohenberg Equation, Spectral Filtering, Bifurcation

\section{Introduction}

The complex Swift-Hohenberg equation (CSHE) was first suggested by Swift and Hohenberg [1] as a simple model for the Rayleigh-Bénard instability of roll waves. This equation models pattern formation arising from an oscillatory instability [2] [3] with a finite wave number at onset and, as such, it admits solutions 
in the form of traveling waves. The CSHE has been widely studied both analytically and numerically [4] [5]. It describes the dynamics of wide-aperture lasers of class A and C close to the peak gain [6]. Besides lasers, the CSHE has been used as a model for other nonlinear optical systems. For instance, photorefractive oscillator, Ker medium, semiconductor laser, and passively mode-locked lasers that allow the generation of self-shaped ultra-short pulses in laser systems [7] are well described by the Complex Swift-Hohenberg order parameter equation. The CSHE is useful to describe solitons propagation in optical systems with linear and nonlinear gain, and spectral filtering such as nonlinear polarization rotation in fiber lasers or communication links including lumped fast absorbers [5]. It seems clear that the generation of more complex impulse can be extremely described by the higher order of the spectral filter.

Initially, one of the generic equations to analyze the dynamics of the dissipative soliton formation in laser systems with a fast saturable absorber is the complex Cubic-quintic Ginzburg-Landau equation (CGLE) model [8] [9]. Nevertheless, the spectral filter of this model is restricted to the second-order term and can only describe a spectral response with a single maximum, which is not the case in many experiments. Indeed, the gain spectrum is usually wide and can have multiple peaks. Thus, in order to make the model more realistic, we need to add other terms of higher-order spectral filtering to the CGLE, leading to the complex Swift-Hohenberg equation [9]. The formation of dissipative soliton in these models has been widely studied in nonlinear dissipative optics. Their properties and conditions of existence have been investigated extensively, from fundamental point of view and due to the clear physical meaning in particular applications [8] [10] [11].

Nonetheless, these studies use purely numerical approaches. Despite the fact that some families of exact solution of the CSHE [12] can be obtained analytically, it is apparent that the CSHE can mainly be analyzed only using computer simulations. Numerical analysis of the CSHE [6] reveals a great variety of patterns and structures such as traveling waves, spiral waves, segregation and competition between stable solutions. In [13] the authors check numerically the validity of the complex Swift-Hohenberg equation for the lasers. However, solving numerically the complex Swift-Hohenberg equation for the two-dimensional solitons, for a given set of parameters and a given initial condition, is an extremely lengthy procedure. To overcome this difficulty, semi-analytical methods based on various physical backgrounds were developed [14]. For instance, in [15] the authors applied the semi-analytical method to investigate soliton propagation and generation of stable moving pulses in one dimension and stable vortex solitons in two dimensions. These alternative theoretical semi-analytical tools can perceive soliton solutions more efficiently in specific domains of the system parameters [9] [14] [16] [17].

Using the collective variable approach, we have expanded the regions of coexistence of 3D dissipative stationary and pulsating solitons in the complex Ginz- 
burg-Landau equation with the cubic-quintic nonlinearity [18]. Recently, with the same approach, we have demonstrated the stationary dissipative solutions of the $2 \mathrm{D}$ complex Swift-Hohenberg equation. Particularly, we mapped the regions of existence of stationary dissipative soliton in the $(v, \varepsilon)$ and $\left(\beta, \gamma_{2}\right)$ planes [5].

Here, our main purpose is to investigate the pulsating solution of the $2 \mathrm{D}$ CSHE using a variational formulation. On the fact that the dynamics of the dissipative solutions are much more complex, and the numerical simulations are extremely tedious tasks, the variational approach is useful to study the ground state since it depends on a trial function and a good set of parameters.

The rest of the paper is organized as follows. We remind in section 2 the collective variable approach and our procedure of determination of the stability domains of the pulsating solutions. Section 3 is devoted to the findings of the $2 \mathrm{D}$ pulsating CSHE solutions. We illustrate the bifurcation behavior and show that they can be stable over a wide range of parameter values. Finally, we summarize with our conclusions in section 4 .

\section{Stability Studies by Collective Coordinates Theory}

In this study, we address the complex Swift-Hohenberg equation in two dimensions. It is helpful to describe soliton propagation in optical systems with linear and nonlinear gain and spectral filtering. As well, the CSHE relates quantitatively as qualitatively many nonlinear effects, which occur during the propagation. This equation is also useful for communication links with lumped fast saturable absorbers or fiber lasers with additive-pulse mode-locking or nonlinear polarization rotation. The CSHE higher order of the spectral filter is extremely essential to analyze the generation of more complex impulse, which makes it preferable in certain situations to the CGLE. The CSHE can be read in this normalized form [8] [19]:

$$
\begin{aligned}
& \psi_{z}-i D \psi_{t t} / 2-i \psi_{r r} / 2-i \gamma|\psi|^{2} \psi-i v|\psi|^{4} \psi \\
& =\delta \psi+\varepsilon|\psi|^{2} \psi+\beta \psi_{t t}+\mu|\psi|^{4} \psi+\gamma_{2} \psi_{t t t}
\end{aligned}
$$

Without the additive term $\gamma_{2} \psi_{t t t}$ this equation is the same as the CGLE one, and here the coefficients $\mu, \delta, \beta, D, \nu, \gamma, \gamma_{2}$ and $\varepsilon$ are real constants. The right-hand-side of Equation (1) contains the dissipative terms: $\gamma_{2}$ represents the higher-order spectral filter term. $\delta, \varepsilon, \beta$ and $\mu$ are the coefficients for linear loss (if negative), nonlinear gain (if positive), spectral filtering (if positive) and saturation of the nonlinear gain (if negative), respectively. The left-hand side holds the conservative terms: namely, $D=+1(-1)$ which is for the anomalous (normal) dispersion propagation regime and $v$ which represents, if negative, the saturation coefficient of the Kerr nonlinearity. $\gamma$ stands for Kerr nonlinearity coefficient. In this present study, the dispersion is anomalous, and $v$ is kept relatively small.

It is clear that the physical meaning of each term of the Equation (1) depends 
on the real problem which must be examined. In optics, when applied to the propagation of the pulses in a laser system, as is the case in our study, $\psi=\psi(r, t, z)$ represents the normalized optical envelope and is a complex function of three real variables. The optical envelope describes not only the pulse as a collective entity localized in time and space but also all other localized or non-localized excitations, such as noise, which are always present in the real system.

The retarded time in the frame moving with the pulse is given by $t$, and $z$ is the propagation distance or the cavity round-trip number. Finally $r\left(r=\sqrt{x^{2}+y^{2}}\right)$ represents the transverse coordinate, taking account of the spatial diffraction effects.

The dynamics of light pulses described by Equation (1) can be stationary [5] and many other types of soliton solutions [20]. In addition, it is a painstaking work to find and classify the different types of the 2D CSHE's solutions. As well, transitions between the solutions occur in the form of sequences of bifurcations [9]. In fact, to the best of our knowledge, there is no analytical solution for the 2D complex Swift-Hohenberg equation. Indeed, researches conducted to date, use namely direct numerical simulations and they fail to reveal more dynamics that are complex. The main and tedious problem is the mapping between the type of solutions and the set of parameters of the equation. To overcome this critical constraint, one option is to use the master equation approach to a certain extent.

We have proved in our previous work [5] the stationary solutions of the 2D CSHE using the collective variable method. We have explained the suitability of this procedure for a systematic search of stability domains for different types of solitons. Here we use the same approach to examine the pulsating soliton of the 2D complex Swift-Hohenberg equation; this has not been done previously as far as we know. Rigorously, we provide an approximate mapping of the regions of existence of stable pulsating solutions in the parameter space of the equation (1), and their dynamics.

In reference to our work [5] [9] [18], we use the Collective variable theory [21] to identify the different types of solutions. The main idea of this approach is to associate collective variables with the pulse parameters of interest for which equations of motion may be derived. To this end, one can decompose the optical field $\psi(r, t, z)$ in the following way:

$$
\psi(r, t, z)=f\left(x_{1}, x_{2}, \cdots, x_{n}, t\right)+q(z, t)
$$

where $f$ the ansatz function is a function of the collective variables $\left(x_{n}\right)$ and is chosen to draw, at best, the configuration of the optical pulse $\psi$. The choice of the ansatz function that introduces the collective variables in the theory is crucial for obtaining solutions with the desired properties and important for the success of the technique. The component $q$ is a residual field that represents all other excitations in the system (noise, radiation, dressing field, etc.).

Subsequently, by neglecting the residual field, one can consider the fact that 
the pulse propagation can be completely characterized by the ansatz function. This approximation is called the bare approximation [21]. As is the case in most practical studies, we use the fact that soliton solutions remain localized and that the exact pulse field is completely characterized by the trial function $(\psi(r, t, z)=f)$. Therefore, we assume that all the pulses are purely Gaussian with spatial and temporal chirp and we do not consider other forms of pulses. This way, we take the following Gaussian function as ansatz function to the success of our approach:

$$
f=A \exp \left(-\frac{t^{2}}{w_{t}^{2}}-\frac{r^{2}}{w_{r}^{2}}+\frac{i}{2} c_{t} t^{2}+\frac{i}{2} c_{r} r^{2}+i p\right)
$$

here the collective variables $A, w_{t}, w_{r}, c_{t}, c_{r}$ and $p$ represent respectively the amplitude, the temporal and spatial widths of the pulse, the chirp along $t$ axis, the spatial chirp and $p$ the global phase. The collective variables evolve along the propagation direction $z$ and the dynamic of the dissipative soliton. In these dynamic and evolutions, the chirps are highly important.

Using the bare approximation to the 2D CSHE (see all the details in [5] [9] [18] [21]) we get the six collective variables that evolve according to the following set of six coupled ordinary differential equations:

$$
\begin{gathered}
\dot{A}=A \delta+\frac{3}{4} A^{3} \varepsilon-\frac{2}{w_{t}^{2}} A \beta+\frac{5}{9} A^{5} \mu-A c_{t} D-2 A c_{r}+3\left(2 c_{t}^{2}-w_{t}^{2} c_{t}^{4}+\frac{3}{w_{t}^{4}}\right) A \gamma_{2}, \\
\dot{w}_{t}=2 w_{t} c_{t} D-\frac{1}{4} w_{t} A^{2} \varepsilon-\frac{2}{9} A^{4} w_{t} \mu+\left(1-w_{t}^{4} c_{t}^{2}\right) \frac{2 \beta}{w_{t}}+\left(w_{t}^{8} c_{t}^{4}-1\right) \frac{12}{w_{t}^{3}} \gamma_{2}, \\
\dot{w}_{r}=4 w_{r} c_{r}-\frac{1}{4} w_{r} A^{2} \varepsilon-\frac{2}{9} A^{4} w_{r} \mu, \\
\dot{c}_{t}=2\left(\frac{1}{w_{t}^{4}}-c_{t}^{2}\right) D-\frac{8}{w_{t}^{2}} c_{t} \beta-\frac{1}{2 w_{t}^{2}} A^{2} \gamma-\frac{4}{9 w_{t}^{2}} A^{4} v+48 c_{t}\left(\frac{1}{w_{t}^{4}}+c_{t}^{2}\right) \gamma_{2}, \\
\dot{p}=2 \beta c_{t}+\frac{3}{4} A^{2} \gamma-\frac{D}{w_{t}^{2}}-\frac{2}{w_{r}^{2}}+\frac{1}{9} A^{4} v-12 c_{t}\left(\frac{1}{w_{t}^{2}}-w_{t}^{2} c_{t}^{2}\right) \gamma_{2}
\end{gathered}
$$

We observe that the CSHE, Equation (1) is reduced to an ordinary differential equation given by the soliton parameters $A, w_{t}, w_{r}, c_{t}, c_{r}$ and $p$. It is clear that these equations give no explicit information with regard to the different solutions of the Equation (1) and their stability. They simply reveal in detail the influence of each parameter of equation CSHE (1) under soliton parameters. In addition, they give us the first idea on the dynamic of the light pulse.

The trial function is suitable for its simplicity, and it makes the procedure of derivation of the variational equations relatively easy. This approach provides the basic parameters of the fixed points (FPs), and a mapping of different types of solutions. The FPs of the system are found by imposing the left-hand side of Equation (4) to be zero ( $\dot{X}=0$ with $X=A, w_{t}, w_{r}, c_{t}, c_{r}, p$ ) [22]. The relation 
$\varepsilon_{s} \approx 2 \sqrt{\delta \mu}$ can estimate the threshold of existence of FPs. If $\varepsilon>\varepsilon_{s}$, we have in general both stable and unstable FPs. The stability of FPs is determined by the analysis of the eigenvalues [22] $\lambda_{j}\left(j=A, w_{t}, w_{r}, c_{t}, c_{r}, p\right)$ of the matrix $M_{i j}=\partial \dot{X}_{i} / \partial X_{j}$.

If the real part of at least one of the eigenvalues $\left(\lambda_{j}\right)$ is positive, the corresponding FP is unstable. The unstable fixed points correspond to the pulsating and non-stationary solutions of the 2D CSHE Equation (1).

If the real parts of all the eigenvalues of the matrix $M_{i j}$ are negative, the corresponding FPs are stable. The stable fixed points correspond to stationary solutions of the 2D CSHE Equation (1). Using the initial condition,

$$
\psi(r, t, 0)=2.86 \exp \left(-\frac{t^{2}}{0.7}-\frac{r^{2}}{1.36}\right)
$$

and investigating the parameter regions situated in the neighbourhood of the parameters $D=\gamma=1, \beta=-0.3, \delta=-0.5, \mu=-0.1$ and $\gamma_{2}=0.05$, we have highlighted the stationary and pulsating solutions in the $(v, \varepsilon)$ plane. For a given set of $v$ and $\varepsilon$ the use of the Newton-Raphson allows finding the corresponding fixed point before determining its stability. The mapping Figure 1 illustrates the result of this rigorous analysis for the range of selected values.

Figure 1 shows the cartography of the solution of the Equation (1). The blue region matches to the fixed points, which represent the stationary solution of the

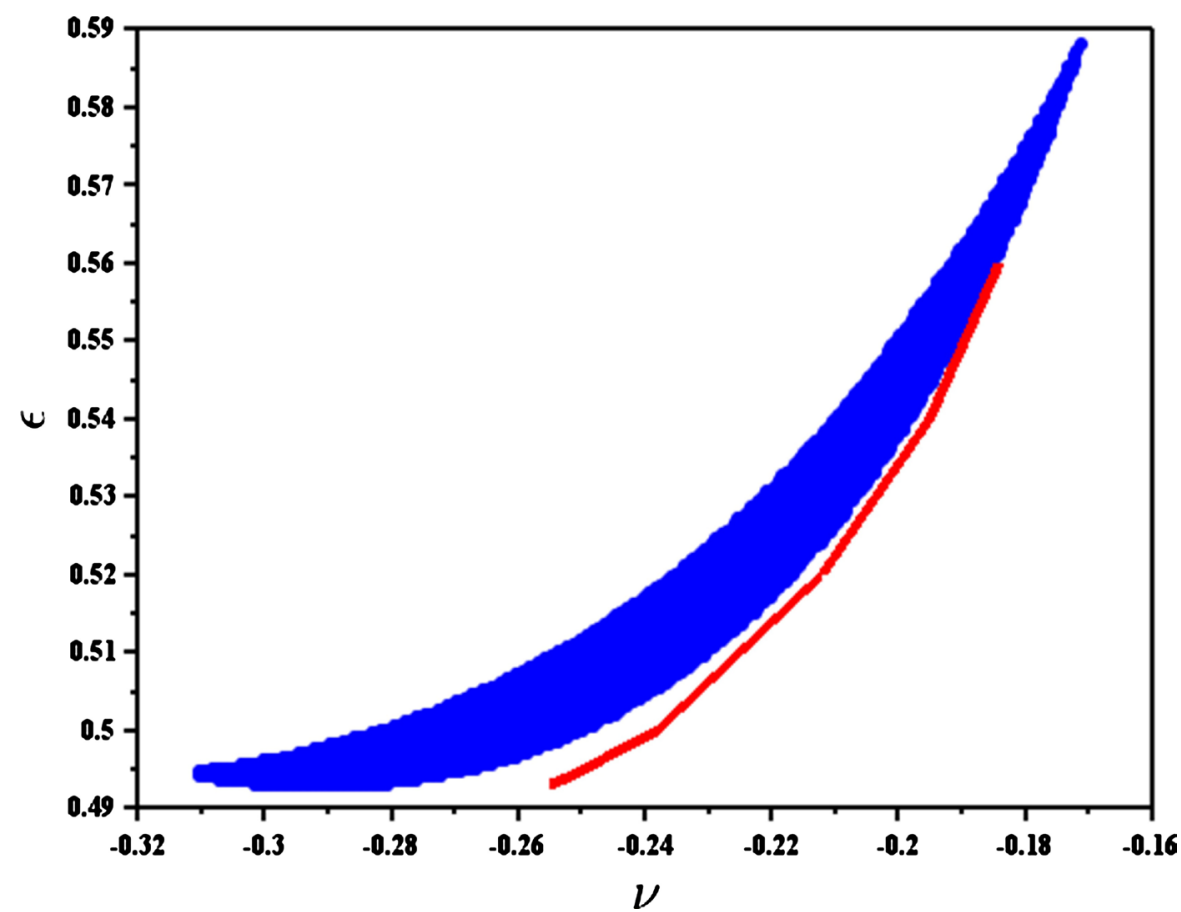

Figure 1. Cartography of the solutions of the 2D complex Swift-Hohenberg equation in the $(v, \varepsilon)$ plane. The stable fixed points regions in blue represent the domain of stationary solitons. The bottom region, between the blue region and the red line corresponds to pulsating solutions. Other CSHE parameters are $D=\gamma=1, \beta=-0.3, \delta=-0.5$, $\mu=-0.1$ and $\gamma_{2}=0.05$. 
2D complex Swift-Hohenberg equation. We studied intensively the distinguishing feature of the stationary solution of the 2D CSHE in [5]. Besides the stationary domain, we have unstable fixed points, which can be divided in two categories: the limit-cycle attractor and the unstable solutions. The limit-cycles region, between the blue region and the red line correspond to pulsating solutions. Indeed close to boundaries of the existence domains of stationary solitons solutions, there is more often an intermediate region in which pulsating solutions can be found. Hereafter we investigate the dynamic of the pulse in the limit-cycle attractor area; the pulsating solutions.

\section{Analysis of the Pulsating Solitons}

The nonlinear dissipative systems are more dynamics than complicated because they include energy exchange with external sources. Besides, they admit pulsating solitons, in contrast to Hamiltonian systems. In [20] Akhmediev and his group presented pulsating solitons of the CGLE and regions of their existence in the five-dimensional parameter space. They also studied the bifurcations within a definite parameter ranges. The authors in [23] carried out pulsating solitons in the existing regions of the CGLE. These results are based on numerical simulations and this problem is not simple as there are several parameters of the CGLE. Except the simulation solutions, some variational formulations have been reported [14]. Recently we addressed the pulsating solitons according to collective variable approach. The existence of pulsating solutions is indeed a general feature of most nonlinear dissipative systems. This behaviour of pulsating soliton can be attributed of limit-cycle attractor; it then possesses inherent stability the same way as stationary stable solutions do.

In order to investigate pulsating soliton in the 2D CSHE, we fix the parameters of the equation as $D=\gamma=1, \beta=-0.3, \delta=-0.5, \mu=-0.1$ and $\gamma_{2}=0.05$. Afterwards, we choose one point between the blue region and the red line (Figure 1) corresponding to pulsating solutions. The related solution to this point is identified by the value of nonlinear gain, $\varepsilon=50$ and the saturation of the Kerr nonlinearity, $v=-0.247$. The dynamic of this pulsating soliton is summarized on the Figure 2. Figure 2(a) shows the evolution of the soliton parameters: amplitude (in red), temporal width (in black) and spatial width (in blue) in the $Z$ direction. In addition, Figure 2(b) and Figure 2(c) are the enlarged views of these parameters. One can clearly notice that the dynamics begin with a transitional phase (according to the initial condition), characterized by small oscillations due to the adjustment of the initial condition. The onset of oscillations is followed by a permanent dynamics that run between two fixed values. The soliton changes its amplitude and widths periodically in the propagation direction, and one pulsation makes one period (see Figure 2). We observe that the soliton periodically changes mostly its widths, while keeping almost constant its peak amplitude. The temporal and spatial widths oscillations can be seen from the evolution plot in the Figure 2(b). We see that the peak amplitude largely 

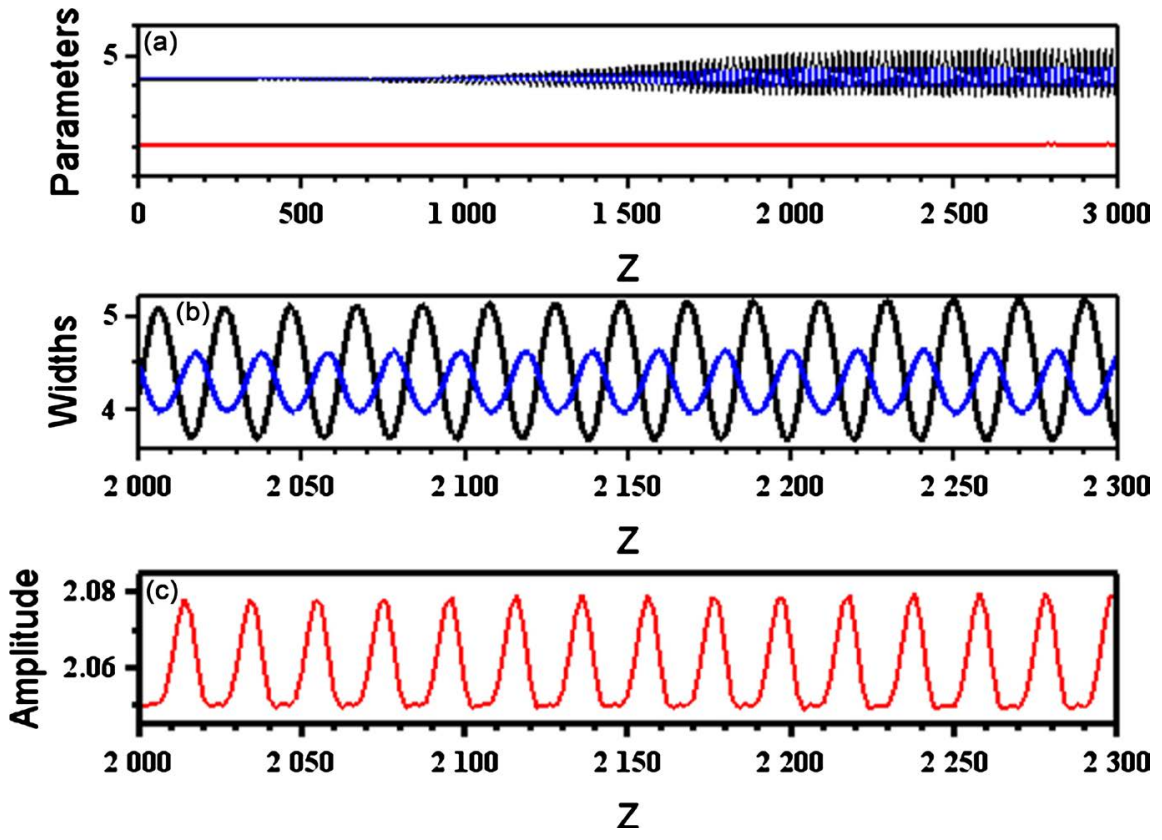

Figure 2. Evolution of pulsating soliton parameters: amplitude (in red), temporal width (in black) and spatial width (in blue), with the use of the collective variable approach and the trial function. Enlarged views of those pulsations are plotted in (b) and (c). The related solution to this point has the value of nonlinear gain to $\varepsilon=50$ and the saturation of the Kerr nonlinearity to $v=-0.247$.

changes periodically rather than the widths. They have the same period, but we can see that the temporal width quickly evolved with respect to the spatial width. The two dynamics are also in opposition of phase. It clearly shows a difference characteristic and oscillation between the temporal and the transverse widths of the $2 \mathrm{D}$ complex Swift-Hohenberg equation.

When the value of the saturation of the Kerr nonlinearity changes from $v=-0.247$ to $v=-0.240$ for the same value of nonlinear gain $\varepsilon=50$, the period of pulsating soliton doubles, that is, the amplitude repeats itself after two pulsations, as shown in Figure 3. The temporal and spatial widths have always presented qualitatively the same behaviors as those in Figure 2 .

However, we notice that the number of pulsations decreases when the value of the saturation of the Kerr nonlinearity goes from $v=-0.247$ to $v=-0.240$. In addition, the amplitude of the oscillations increases.

The analysis of these two figures (Figure 2 and Figure 3) reveals that the saturation of the Kerr nonlinearity plays an important part in the internal dynamic of the 2D complex Swift-Hohenberg equation pulsating soliton.

The parameter $v$ influences both qualitatively and quantitatively the pulsations of the soliton. As well, the saturation of the Kerr nonlinearity plays a key role by changing a single periodic pulsating soliton to a double period pulsating soliton. Figure 4 shows clearly the evolution of the pulsating soliton parameters for a given set of parameters corresponding to the pulsating domain. We change the nonlinear gain from $\varepsilon=50$ to $\varepsilon=54$ and the saturation of the Kerr 


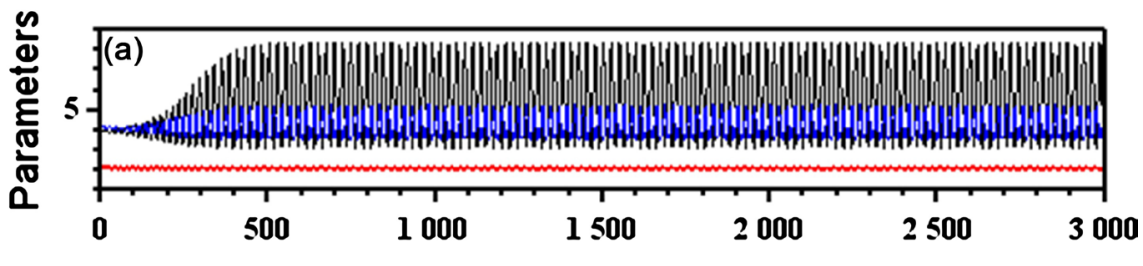

Z

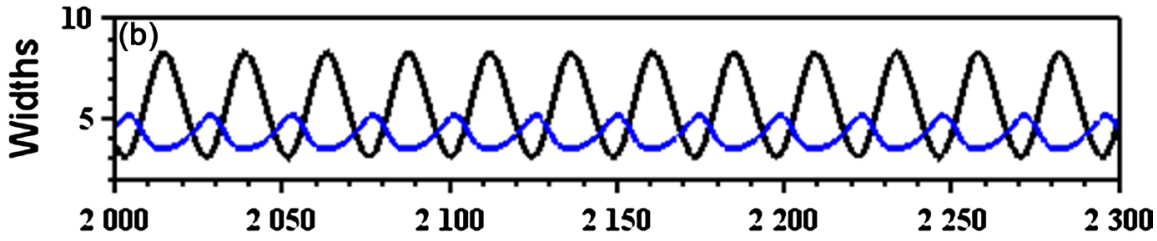

Z

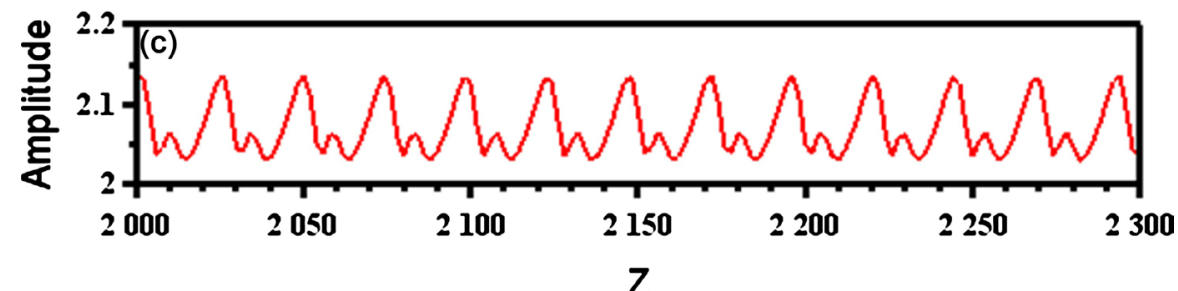

Figure 3. Evolution of pulsating soliton parameters: amplitude (in red), temporal width (in black) and spatial width (in blue), with the use of the collective variable approach and the trial function. Enlarged views of those pulsations are plotted in (b) and (c). The related solution to this point has the value of nonlinear gain to $\varepsilon=50$ and the saturation of the Kerr nonlinearity to $v=-0.240$.
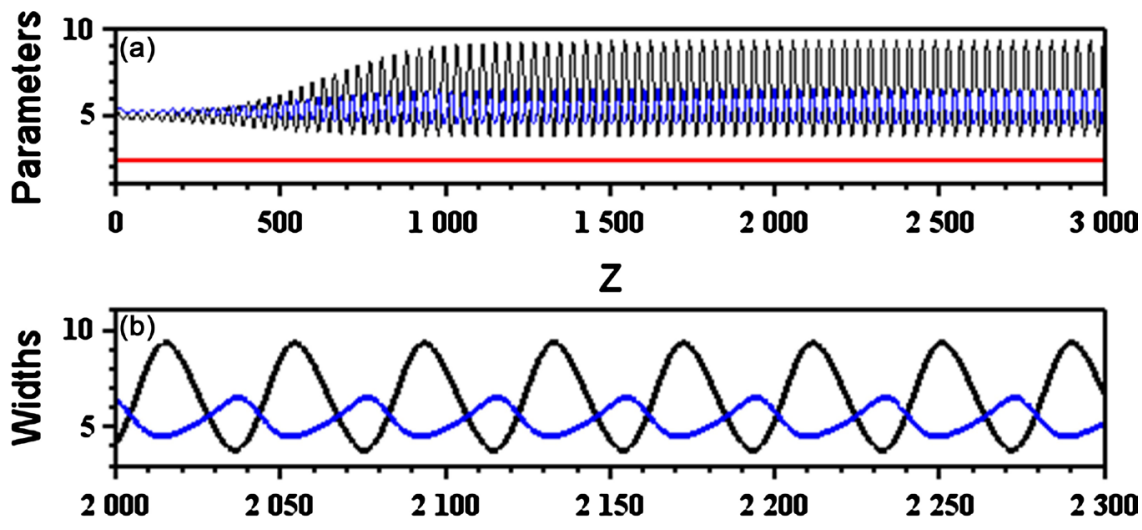

Z

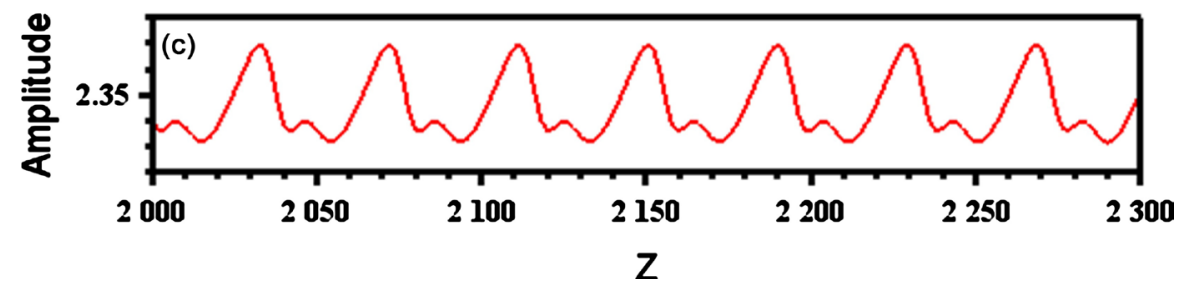

Figure 4. Evolution of pulsating soliton parameters: amplitude (in red), temporal width (in black) and spatial width (in blue), with the use of the collective variable approach and the trial function. Enlarged views of those pulsations are plotted in (b) and (c). The related solution to this point has the value of nonlinear gain to $\varepsilon=54$ and the saturation of the Kerr nonlinearity to $v=-0.196$. 
nonlinearity from $v=-0.240$ to $v=-0.196$. Figure 4 (a) shows the evolution of the soliton parameters: amplitude (in red), temporal width (in black) and spatial width (in blue) in the $z$ direction, and Figure 4(b) and Figure 4(c) are the enlarged views of these parameters. For these values of the nonlinear gain and the saturation of the Kerr nonlinearity, we observe that the number of pulsations of the widths and the amplitudes decreases considerably. At the same time, their amplitudes of the oscillations increase.

The total energy plays a whole role in the study of the complex dissipative systems. Here it gives us the main information about the soliton dynamics. It is not conserved but evolves in accordance with the so-called balance equation. When a pulsating solution is reached, the total energy is an oscillating function of the propagation distance. Thus, all the solitons parameters (amplitude, widths, chirp ...) stay pulsating throughout propagation, as has been seen in Figure 2, Figure 3 and Figure 4. The total energy of the system also has the same dynamic, which does not change at all, during propagation for these values considered. In order to highlight the action of the energy of the pulsating solitons, we observed its evolution upon propagation.

We plotted the dynamics of the energy for $\varepsilon=50$ and $v=-0.240$ (Figure 5), for $\varepsilon=54$ and $v=-0.196$ (Figure 6), the other parameters remain constant. It clearly appears that the group dynamics are mostly similar to those of the evolution of the pulsating wave parameters (amplitude and widths). The pulsating properties of double period oscillation can be seen obviously. For larger values of parameters ( $\varepsilon$ from 50 to 54 , and $v$ from -0.240 to -0.196 ) the
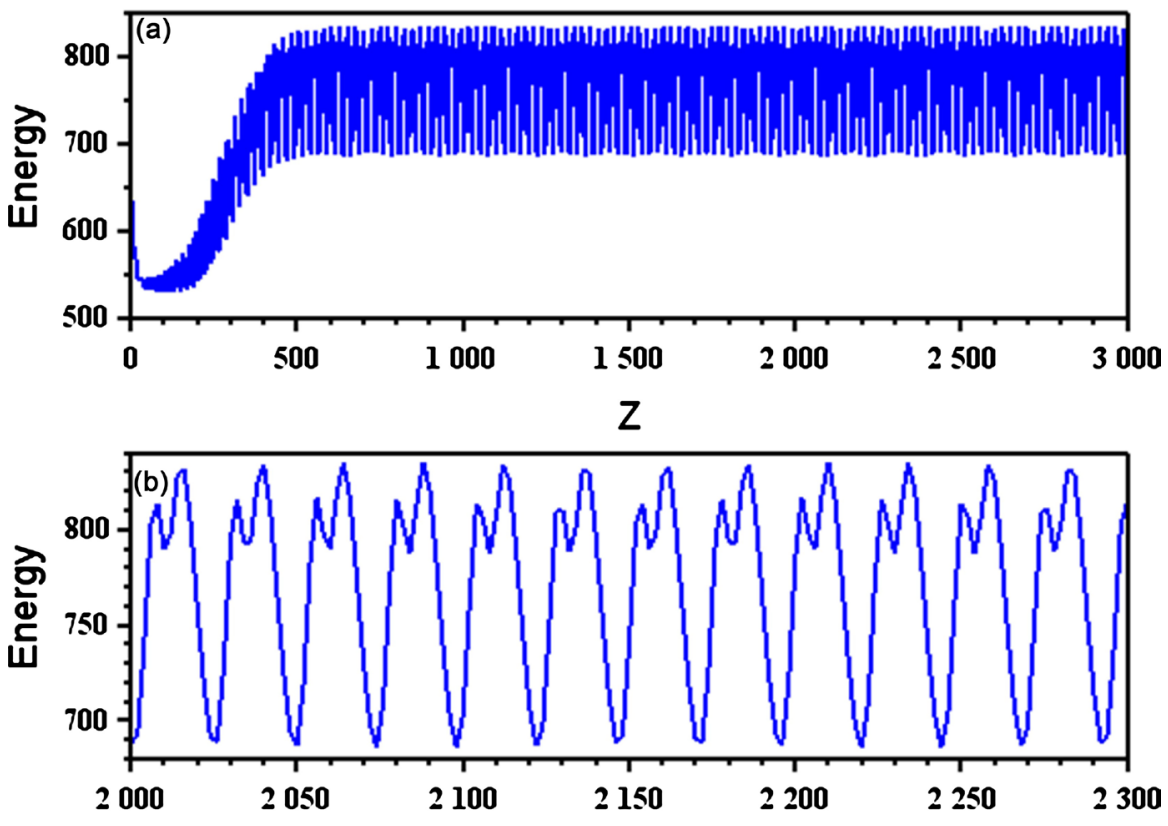

$Z$

Figure 5. Evolution of the total pulse energy, enlarged view of this pulsation is plotted bottom with $D=\gamma=1, \quad \beta=-0.3, \delta=-0.5, \quad \mu=-0.1, \quad \gamma_{2}=0.05, \quad \varepsilon=50$ and $v=-0.240$. 
pulse wave remain pulsating, but the pulse energy is much higher (Figure 6) than before (Figure 5). On the other hand, this fact greatly affects the quality and the period of oscillations. The results show that an increase of the nonlinear gain and the saturation of the Kerr nonlinearity induce pulsating solitons with significant amounts of energy. As shown below in Figure 7 the temporal profile
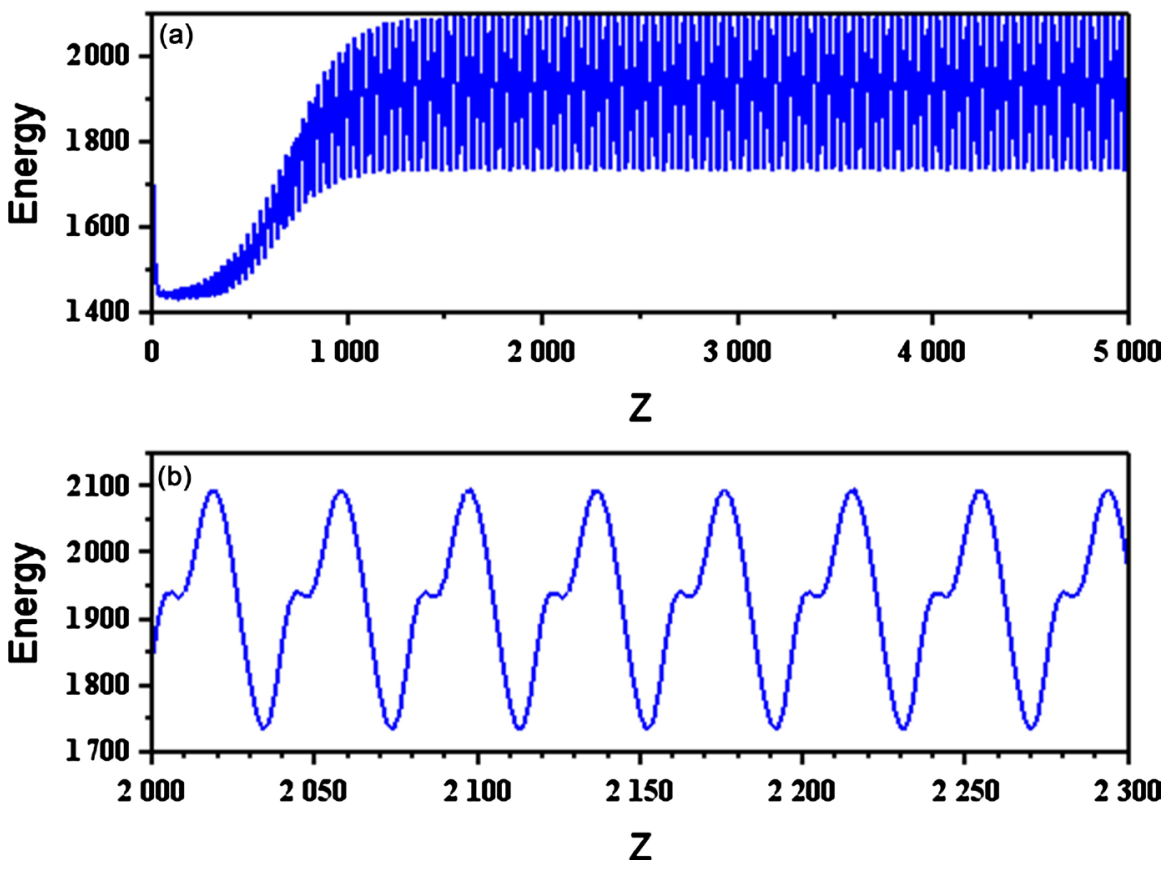

Figure 6. Evolution of the total pulse energy, enlarged view of this pulsation is plotted bottom with $D=\gamma=1, \quad \beta=-0.3, \delta=-0.5, \quad \mu=-0.1, \quad \gamma_{2}=0.05, \varepsilon=54$ and $v=-0.196$.

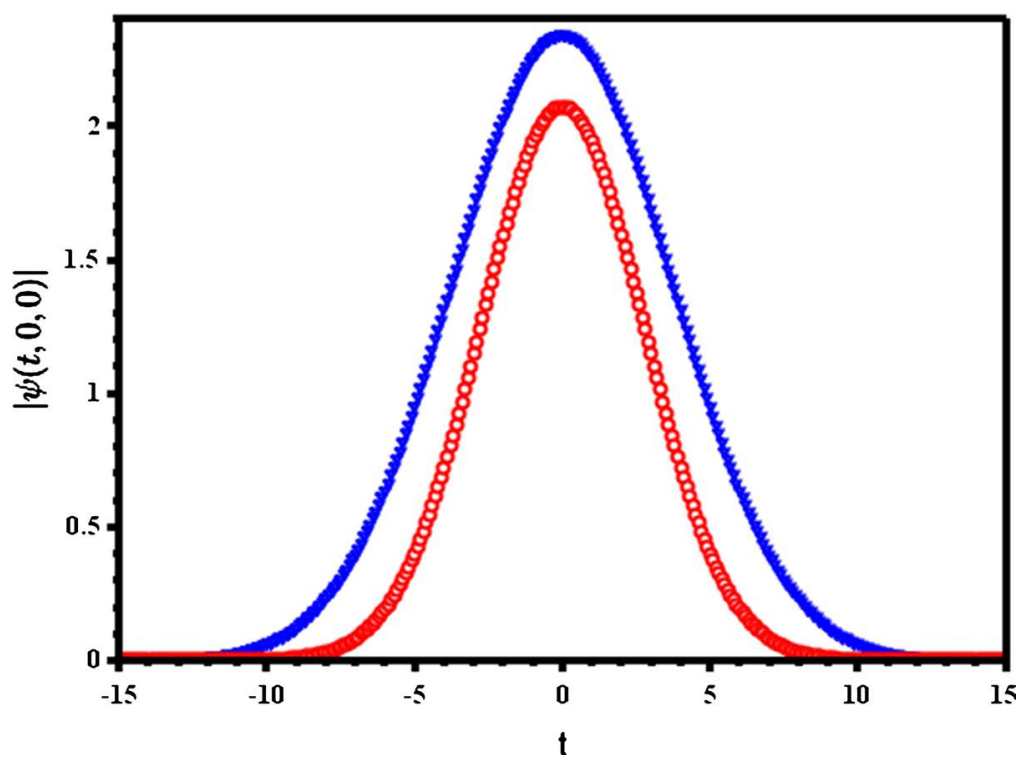

Figure 7. The temporal profiles of the total pulse pulsating solitons. In blue for $\varepsilon=54$ and $v=-0.196$, in red $\varepsilon=50$ and $v=-0.240$. Other CSHE parameters are $D=\gamma=1, \quad \beta=-0.3, \delta=-0.5, \quad \mu=-0.1$ and $\gamma_{2}=0.05$. 
also becomes more important, a characteristic sign of a larger impulse.

During this present study, it distinctly appeared that the saturation of the Kerr nonlinearity $v$ has a real impact on the dynamics of the pulsating solitons. Changing this parameter and keeping the other parameters constant, the stationary soliton becomes pulsating with one period, and then the pulsating soliton bifurcates to double period pulsations. To illustrate this phenomenon, we plot in the Figure 8 a bifurcation diagram obtained when varying the saturation of the Kerr nonlinearity $v$ from -0.26 to -0.235 , while keeping the rest of parameters (see Figure 8) fixed.

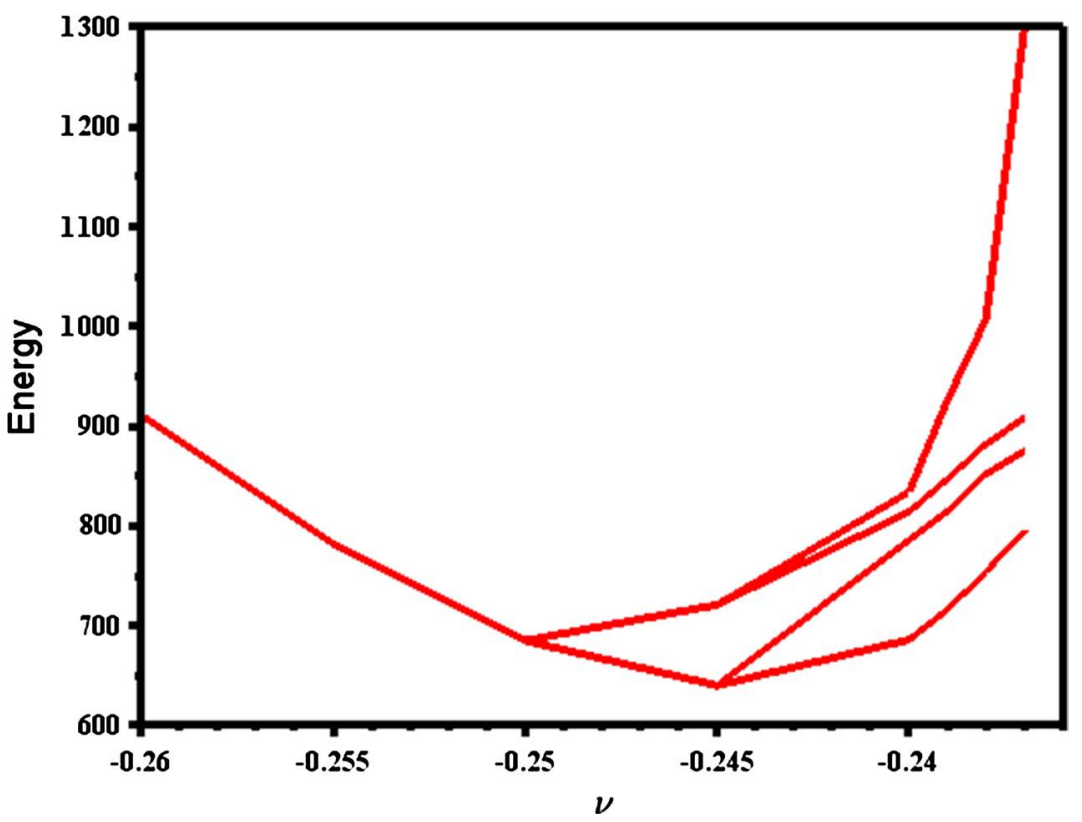

Figure 8. Bifurcation diagram of the dissipative pulsating solitons. Other CSHE parameters are $D=\gamma=1, \beta=-0.3, \delta=-0.5, \mu=-0.1, \varepsilon=50$ and $\gamma_{2}=0.05$.

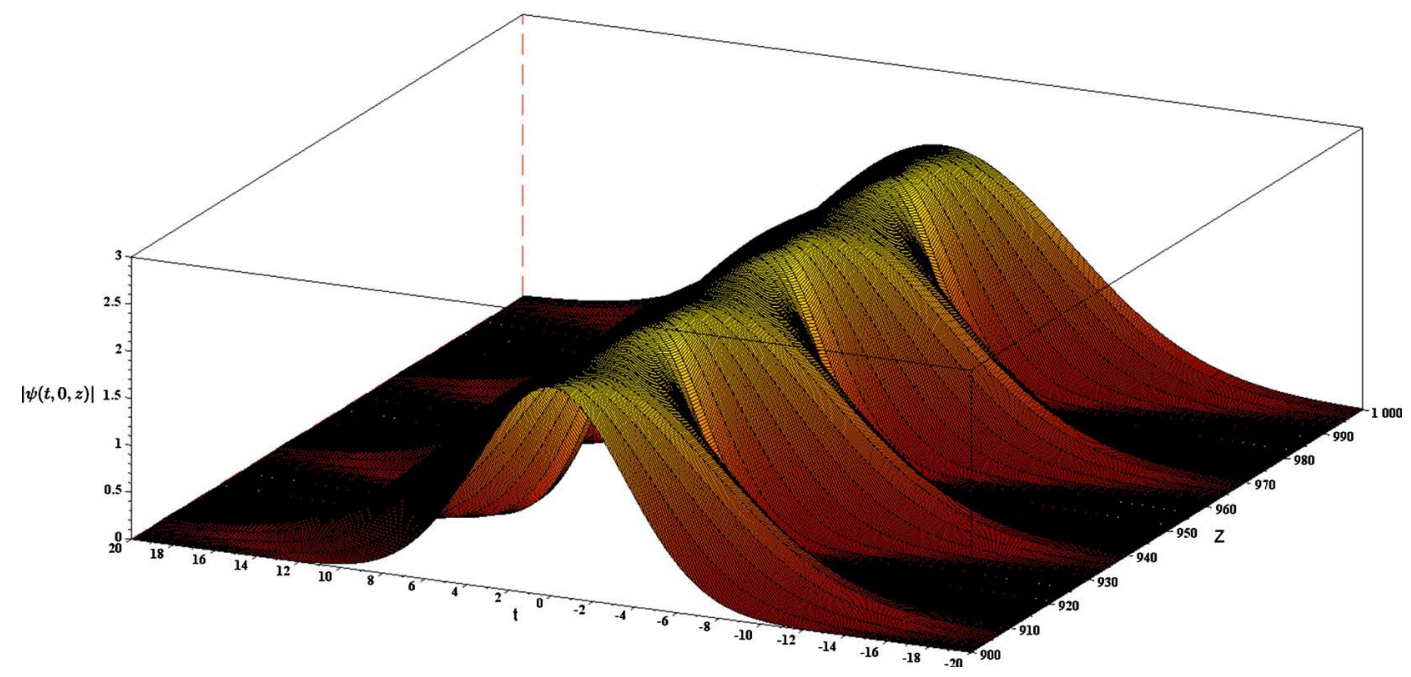

Figure 9. Evolution of pulsating soliton for $\varepsilon=52$ and $v=-0.213$. Other CSHE parameters are $D=\gamma=1, \beta=-0.3$, $\delta=-0.5, \mu=-0.1$ and $\gamma_{2}=0.05$. 
For a given set of the saturation of the Kerr nonlinearity, the curve (Figure 8) represents a local maximum or minimum of the total energy. We observe that the total energy leads to a pulsation mode whose spectrum contains two main frequencies. When the value of parameter $v$ is smaller -0.25 the total energy of the soliton has a single value (maximum equal to minimum), which corresponds to stationary soliton. When the saturation of the Kerr nonlinearity $v$ changes from -0.25 to -0.245 a first bifurcation occurs, which is related to the onset of pulsations with single frequency, the soliton presents a single period pulsating solution, and oscillates between one maximum and one minimum. The corresponding profile of such pulsating soliton is shown in Figure 9 to resume the dynamic. For the values of the parameter $v$ greater than $-0.245 \mathrm{a}$ second bifurcation occurs again. Thereafter the pulsating soliton oscillates with two frequencies, presents double periods and changes between two maximum and two minimum.

\section{Conclusion}

We have carried out the dynamical behavior of pulsating solitons in the two-dimensional Complex Swift-Hohenberg equation. Thanks to collective variable approach, the regions of coexistence of pulsating dissipative solitons are obtained. This semi-analytical method is a useful tool to predict pulsating solutions when a suitable trial function is chosen in physical systems of high dimension. The detailed analysis shows that the nonlinear gain and the saturation of the Kerr nonlinearity influence both qualitatively and quantitatively the pulsations of the pulsating solitons. The complete bifurcation diagram has been obtained for a definite range of the saturation of the Kerr nonlinearity values. The diagram reveals that when the saturation of the Kerr nonlinearity increases, one period pulsating solution bifurcates to double period pulsations. This study reveals the rich dynamics of the pulsating solutions in the $2 \mathrm{D}$ CSHE and could be completed by the analysis of their properties. It can also help to describe specific aspects that occur in wide-aperture laser cavity.

\section{Conflicts of Interest}

The authors declare no conflicts of interest regarding the publication of this paper.

\section{References}

[1] Swift, J. and Hohenberg, P.C. (1977) Hydrodynamic Fluctuations at the Convective Instability. Physics Review A, 15, 319-328 https://doi.org/10.1103/PhysRevA.15.319

[2] Bestehorn, M. and Haken, H. (1990) Traveling Waves and Pulses in a Two-Dimensional Large-Aspect-Ratio System. Physics Review A, 42, 7195.

https://doi.org/10.1103/PhysRevA.42.7195

[3] Malomed, B.A. (1984) Nonlinear Waves in Nonequilibrium Systems of the Oscillatory Type, Part I. Zeitschrift für Physik B Condensed Matter, 55, 241-248.

https://doi.org/10.1007/BF01329018

[4] Peletier, L.A. and Williams, J.F. (2007) Some Canonical Bifurcations in the Swift-Ho- 
henberg Equation. SIAM Journal on Applied Dynamical Systems, 6, 208-235. https://doi.org/10.1137/050647232

[5] Yoboue, P., Diby, A., Asseu, O. and Kamagate, A. (2016) Stability of Dissipative Optical Solitons in the 2D Complex Swift-Hohenberg Equation. International Journal of Physics, 4, 78-84.

[6] Hoyuelos, M. (2005) Numerical Study of the Vector Complex Swift-Hohenberg Equation. Physica D: Nonlinear Phenomena, 223, 174-179.

https://doi.org/10.1016/j.physd.2006.09.002

[7] Ippen, E.P. (1994) Principles of Passive Mode Locking. Applied Physics B, 58, 159-170. https://doi.org/10.1007/BF01081309

[8] Akhmediev, N. and Ankiewicz, A. (2005) Dissipative Solitons. Springer, Heidelberg.

[9] Kamagaté, A., Grelu, Ph., Tchofo-Dinda, P., Soto-Crespo, J.M. and Akhmediev, N. (2009) Stationary and Pulsating Dissipative Light Bullets from a Collective Variable Approach. Physical Review E, 79, 026609.

https://doi.org/10.1103/PhysRevE.79.026609

[10] Akhmediev, N. and Ankiewicz, A. (2008) Dissipative Solitons: From Optics to Biology and Medicine. Springer, Heidelberg.

[11] Khairudin, N.I., Abdullah, F.A. and Hassan, Y.A. (2016) Stability of the Fixed Points of the Complex Swift-Hohenberg Equation. Journal of Physics: Conference Series, 693, 012003. https://doi:10.1088/1742-6596/693/1/012003

[12] Maruno, K., Ankiewicz, A. and Akhmediev, N. (2003) Exact Soliton Solutions of the One-Dimensional Complex Swift-Hohenberg Equation. Physica D, 176, 44-66. https://doi.org/10.1016/S0167-2789(02)00708-X

[13] Pedrosa, J., Hoyuelos, J.M. and Martel, C. (2008) Numerical Validation of the Complex Swift-Hohenberg Equation for Lasers. The European Physical Journal B, 66, 525-530. https://doi.org/10.1140/epjb/e2008-00457-5

[14] Arnous, A.H., Mirzazadeh, M., Moshokoa, S., Medhekar, S., Zhou, Q., Mahmood, M.F., Biswas, A. and Belic, M. (2015) Solitons in Optical Metamaterials with Trial Solution Approach and Backlund Transform of Riccati Equation. Journal and Computational and Theoretical Nanoscience, 12, 5940-5948.

https://doi.org/10.1166/jctn.2015.4739

[15] Wang, H. and Yanti, L. (2011) An Efficient Numerical Method for the Quintic Complex Swift-Hohenberg Equation. Numerical Mathematics. Theory, Methods and Applications, 4, 237-254.

[16] Soto-Crespo, J.M., Akhmediev, N.N. and Afanasjev, V.V. (1996) Stability of the Pulselike Solutions of the Quintic Complex Ginzburg-Landau Equation. Journal of the Optical Society of America B, 13, 1439-1449. https://doi.org/10.1364/JOSAB.13.001439

[17] Skarla, V. and Aleksic, N. (2006) Stability Criterion for Dissipative Soliton Solutions of the One- , Two- , and Three-Dimensional Complex Cubic-Quintic Ginzburg-Landau Equations. Physical Review Letters, 96, 013903. https://doi.org/10.1103/PhysRevLett.96.013903

[18] Asseu, O., Diby, A., Yoboué, P. and Kamagaté, A. (2016) Spatio-Temporal Pulsating Dissipative Solitons through Collective Variable Methods. Journal of Applied Mathematics and Physics, 4, 1032-1041. https://doi.org/10.4236/jamp.2016.46108

[19] Akhmediev, N. and Ankiewicz, A. (2001) Solitons of the Complex Ginzburg-Landau Equation. Springer, Berlin. https://doi.org/10.1007/978-3-540-44582-1_12

[20] Akhmediev, N., Soto-Crespo, J.M. and Town, G. (2001) Pulsating Solitons, Chaotic 
Solitons, Period Doubling, and Pulse Coexistence in Mode-Locked Lasers: Complex Ginzburg-Landau Equation Approach. Physical Review E, 63, 056602. https://doi.org/10.1103/physreve.63.056602

[21] Tchofo-Dinda, P., Moubissi, A.B. and Nakkeeran, K. (2001) Collective Variable Theory for Optical Solitons in Fibers. Physical Review E, 64, 016608. https://doi.org/10.1103/PhysRevE.64.016608

[22] Tsoy, E.N. and Akhmediev, N. (2005) Bifurcations from Stationary to Pulsating Solitons in the Cubic-Quintic Complex Ginzburg-Landau Equation. Physics Letters A, 343, 417-422. https://doi.org/10.1016/j.physleta.2005.05.102

[23] Wu, L., Guo, Z.J. and Song, L.-J. (2010) Properties of Pulsating Solitons in Dissipative Systems. Chinese Physics B, 19, 080512.

https://doi.org/10.1088/1674-1056/19/8/080512 